Relations industrielles

Industrial Relations

\title{
Vouloir et savoir négocier...
}

\section{Gérard Tremblay}

Volume 1, numéro 10, juin 1946

URI : https://id.erudit.org/iderudit/1023959ar

DOI : https://doi.org/10.7202/1023959ar

Aller au sommaire du numéro

\section{Éditeur(s)}

Département des relations industrielles de l’Université Laval

\section{ISSN}

0034-379X (imprimé)

1703-8138 (numérique)

Découvrir la revue

Citer cet article

Tremblay, G. (1946). Vouloir et savoir négocier... Relations industrielles / Industrial Relations, 1(10), 2-3. https://doi.org/10.7202/1023959ar

Tous droits réservés ( Département des relations industrielles de l’Université Laval, 1946
Ce document est protégé par la loi sur le droit d'auteur. L’utilisation des services d'Érudit (y compris la reproduction) est assujettie à sa politique d'utilisation que vous pouvez consulter en ligne.

https://apropos.erudit.org/fr/usagers/politique-dutilisation/ 


\section{VOULOIR ET SAVOIR NÉGOCIER ...}

La législation du travail a subi une orientation nouvelle au Canada, depuis 1944. L'adoption des règlements fédéraux relatifs aux relations ouvrières en temps de guerre (C.P. 1003) et de la Loi des relations ouvrières dans le Québec a mis en lumière des principes déjà agréés par l'opinion publique, à savoir :

a) que l'association ouvrière est de bien commun ;

b) que la négociation collective des conditions de travail doit constituer une procédure obligatoire.

Nous nous demandons toutefois si patrons et ouvriers se rendent réellement compte des responsabilités sociales accrues qui leur échoient.

La liberté syndicale a été conquise ; le droit d'association est protégé explicitement par l'interdicton des pratiques anti-syndicales. A ce droit activement protégé par l'Etat correspond un devoir précis pour ceux qui en usent. Les salariés doivent se former en associations, mais en développant parallèlement chez eux un grand sens de responsabilité sociale. L'individu ne disparaît pas mais son individualisme s'atténue. Il y perd en liberté mais il y gagne en force en s'intégrant à un groupe social activiste. Ses actions n'ont plus seulement une signification individuelle : elles ont une répercussion sociale conditionnée par l'importance du milieu. L'ouvrier non syndiqué, individualiste, qui réclame pour lui-même un relèvement de salaire et quitte son travail faute de satisfaction ne cause qu'un léger tort à la société. Le même ouvrier, s'il se syndique et devient orateur habile, peut amener tous ses confrères de travail à faire la grève. Ses actes le dépassent; il peut, en maniant imprudemment son autorité sur un groupe, mettre à mal tout un secteur de l'activité économique.

Le patronat a des responsabilités identiques. L'employeur ne doit pas bouder la législation sur la liberté syndicale, mais plutôt s'y adapter en toute sincérité. Négociation collective implique collaboration du Capital et du Travail. Les actes d'un employeur échappent à la norme individualiste ; ils ont une portée de plus en plus sociale. Dans les relations industrielles, l'employeur ne traite plus avec ses ouvriers, mais avec leur mandataire. Il doit voir dans celui-ci le représentant de tout un facteur de la production et non plus un ouvrier. Psychologiquement, les attitudes doivent se modifier. L'employeur doit se rappeler que la liberté d'expression et d'action qu'il pouvait se permettre à l'endroit de l'ouvrier isolé n'est maintenant plus de mise et qu'il y faut mettre des formes : on ne traite pas un groupe comme on traite un individu.

Représentants patronaux et ouvriers, mis par la loi dans l'obligation de négocier de bonne foi, se rendent compte de l'éminence de leur tâche, de sa nature, de son climat. Ils doivent d'abord "vouloir " négocier. Il semble curieux d'insister sur l'élément volonté dans la négociation collective. Négocier est une chose ardue, pénible, irritante; il faut y mettre non seulement de l'intelligence, mais de la compréhension, de la patience, de la fermeté. Le patron trouve plus facile de dire non ou de tenter d'imposer d'autorité son point de vue ; de son côté, le mandataire ouvrier aime mieux couper court à la discussion et recommander une solution de force, la grève, si tant est qu'elle est parfois permise ; ou encore, les deux parties jettent un œil du côté des services publics de conciliation et d'arbitrage : c'est tellement plus commode et moins ennuyeux! Si bien qu'aujourd'hui, on réclame le concours de l'Etat pour régler la moindre difficulté ỏu le moindre litige. N'est-ce pas démissionner de ses responsabilités? N'est-ce pas arracher l'Etat à son rôle supplétif et user son autorité à régler des vétilles ? L'Etat ne doit pas être propagandiste ou agent d'affaires; encore une fois, il doit compléter l'action des individus et des groupes et non pas s'y substituer.

Négocier de bonne foi est salutaire pour les parties à un accord. Cette procédure leur permet d'exposer leurs points de vue, de composer leurs différends, de compenser les concessions mutuelles, de se mieux connaître et en fin de compte, de mieux s'entendre en toute équité.

Vouloir négocier est bien mais il faut aussi savoir négocier un accord. Des méthodes fondées sur la théorie et l'expérience sont déjà admises et doivent être développées. Voici quelques normes pouvant servir aux représentants des salariés :

$1^{\circ}$ Formation démocratique du syndicat et de ses officiers.

$2^{\circ}$ Respect des principes et procédures établis par la Loi des relations ouvrières.

$3^{\circ}$ Préparation soignée du projet de convention collective, préalablement approuvé par la majorité des syndiqués intéressés.

$4^{\circ}$ Choix d'agents négociateurs intelligents, bien au fait des techniques de l'industrie, polis, psychologues, patients, énergiques et honnêtes.

$5^{\circ}$ L'agent négociateur doit connaître l'industrie dont il discute : procédés de fabrication ; machinerie utilisée; conditions d'hygiène et de sécurité; catégories professionnelles diverses; base du taux de rémunération; systèmes de primes au rendement ; salaires payés dans les industries comparables; indice du cout de la vie ; condition du marché.

$6^{\circ}$ Dès que la négociation est terminée et que les parties au débat en sont venues à un accord acceptable, les représentants syndicaux doivent présenter celui-ci à leurs confrères et tenter loyalement de le faire accepter.

$7^{\circ}$ Le représentant syndical ne doit pas être neutre à l'égard d'un projet d'accord qu'il a obtenu ; il doit en montrer les avantages et les inconvénients, mais il ne doit pas hésiter à jeter le poids de son influence en faveur 
d'une acceptation. Le représentant syndical est un mandataire, mais aussi un chef et un chef doit conseiller, diriger.

$8^{\circ}$ Une convention collective peut être revisée chaque année ; si elle n'est pas parfaite, il y aura lieu de l'améliorer ultérieurement.

L'employeur, de son côté, ne peut entreprendre de négociations collectives avec ses ouvriers sans préparation ; c'est pourquoi nous nous permettons de lui dire :

$1^{\circ}$ Etudiez les lois qui régissent les relations patronales-ouvrières; pour en discuter pertinemment, vous devez les connaître.

$\mathscr{2}^{\circ}$ Enquêtez sur les conditions de vie de vos ouvriers.

$\mathfrak{S}^{\circ}$ Préparez des statistiques sur les salaires moyens par catégories professionnelles, pour l'ensemble de votre personnel.

$4^{\circ}$ Préparez l'état financier de l'entreprise au cas où il serait nécessaire d'y référer.

$5^{\circ}$ Colligez des renseignements sur les taux de salaires dans les industries comparables.

$6^{\circ} \mathrm{Ne}$ refusez jamais immédiatement une requête ; mettez-la à l'étude, acceptez-la ou repoussez-la en tout ou en partie avec arguments à l'appui, à une réunion subséquente.

$7^{\circ} \mathrm{Si}$ on vous soumet un projet de convention, recevezle aimablement. Faites votre contre-projet, si nécessaire.

$8^{\circ}$ Vos ouvriers sont vos collaborateurs. Ils ont été la source de votre fortune passée ; soyez-leur sympathique : il est naturel qu'ils veuillent améliorer leurs conditions de vie.

$9^{\circ}$ Avant de refuser une augmentation des taux de salaires, étudiez votre entreprise. Est-elle bien organisée? Votre outillage ne peut-il être amélioré? L'agencement des procédés de fabrication est-il rationnel? Ne pouvez-vous pas faire des économies? Vous occupez-vous d'accroitre vos marchés? Et la routine ?... En bref, il ne faut pas qu'un refus d'amélioration des conditions de travail soit attribuable à vos déficiences et à vos manquements.

$10^{\circ}$ N'oubliez pas que compréhension, politesse et courtoisie sont toujours de mise.

Patrons et ouvriers doivent done tenir pour certain qu'il y a une technique de la négociation, qu'elle ne s'improvise pas mais s'acquiert par l'étude et la pratique du métier. Si cette technique était mieux possédée, nous aurions moins de différends du travail, moins de recours à l'ingérence de l'État et plus de justice sociale.

Le Département des relations industrielles s'est rendu compte de l'urgence de son humble collaboration dans ce domaine. Déjà, depuis deux ans, en plus de ses cours universitaires réguliers, il a organisé de nombreuses conférences publiques, un congrès des relations industrielles et deux sessions intensives de quin ze jours. Il continuera dans cette voie où le suivront volontiers tous ceux qui veulent que les relations entre le Capital et le Travail s'élaborent sous le signe de l'intelligence et de la bonne foi.

Gerard Tremblay.
MEMOIRES ET THĖSES

SUR LES RELATIONS INDUSTRIELLES

PREESENTEES POUR L'OBTENTION

DU BACCALAUREAT ET DE LA MAÎTRISE

EN SCIENCES SOCIALES.

\section{MÉMOIRES}

André Parent

" Les clauses de sécurité syndicale dans les conventions collectives de la province de Québec. ")

Jean-Paul Ferland

" Analyse des conventions collectives négociées par la Fédération nationale des travailleurs de la pulpe et du papier. "

Jean-Mathieu Cantín

"L'employeur et le problème du travail. ".

Charles Bélanger

( Les salaires minima des artisans de la construction à Québec (1925-1945). "

Jacques Coté

"Vue d'ensemble des principaux éléments de monopole dans l'industrie du papier à journal au Canada. "

Henri Legaré, o.m.i.

"Congés payés dans la province de Québec. "

Edouard-A. Tardieu

"Le problème des salaires. "

JAMES Hodgson

" Introduction historique à l'étude de l'industrie de la chaussure."

Gilles Lavoie

"Les pratiques interdites dans les relations du travail. ")

\section{THÈSES}

Henri Legaré, o.m.i.

" Le rôle social du prêtre à la lumière des documents pontificaux. ")

Andre Després

" L'opinion publique et les relations industrielles. "

Monique Boucher

"Le travail féminin dans la province de Québec. "

JAMES Hodgson

"L'industrie de la chaussure au Canada. " 